PROCEEDINGS OF THE

AMERICAN MATHEMATICAL SOCIETY

Volume 137, Number 3, March 2009, Pages 1021-1024

S 0002-9939(08)09596-8

Article electronically published on September 25, 2008

\title{
PLEIJEL'S NODAL DOMAIN THEOREM FOR FREE MEMBRANES
}

\author{
IOSIF POLTEROVICH
}

(Communicated by Walter Craig)

\begin{abstract}
We prove an analogue of Pleijel's nodal domain theorem for piecewise analytic planar domains with Neumann boundary conditions. This confirms a conjecture made by Pleijel in 1956. The proof is a combination of Pleijel's original approach and an estimate due to Toth and Zelditch for the number of boundary zeros of Neumann eigenfunctions.
\end{abstract}

\section{INTRODUCTION}

Let $\Omega \subset \mathbb{R}^{2}$ be a bounded planar domain. Let $0 \leq \lambda_{1}<\lambda_{2} \leq \cdots \leq \lambda_{k} \leq \ldots$ be the eigenvalues of the Laplacian on $\Omega$ with either Dirichlet or Neumann boundary conditions, and $\phi_{1}, \phi_{2}, \ldots, \phi_{k}, \ldots$ be an orthonormal basis of eigenfunctions: $\Delta \phi_{k}=\lambda_{k} \phi_{k}, k=1,2, \ldots$ We assume that the boundary $\partial \Omega$ is sufficiently regular (say, piecewise smooth). In the Neumann case we also assume throughout the paper that the internal cone condition [EE, chapter V.4] is satisfied at the corners in order to ensure that the spectrum is discrete. According to the classical Courant's nodal domain theorem [Co (see also [CH, p. 452]), the number $n_{k}$ of nodal domains of the eigenfunction $\phi_{k}$ is at most $k$. In 1956, Pleijel [Pl] showed that for planar domains with Dirichlet boundary conditions Courant's bound can be asymptotically improved:

$$
\limsup _{k \rightarrow \infty} \frac{n_{k}}{k} \leq \frac{4}{j_{1}^{2}} \approx 0.691 \ldots,
$$

where $j_{1} \approx 2.4$ is the first zero of the Bessel function $J_{0}$. In particular, this implies that the equality in Courant's theorem is attained only for a finite number of eigenfunctions. Note that in the 1-dimensional case, the eigenfunction $\phi_{k}$ has exactly $k$ nodal domains for any $k=1,2, \ldots$.

The proof of the estimate (1.1) is an application of the Faber-Krahn inequality for the first eigenvalue ([Fa,, $\mathrm{Kr}$; see also $[\mathrm{Ch}$, p. 87]). In [Pl, section 7], Pleijel writes regarding the inequality (1.1) that "...it seems highly probable that the result... is also true for free membranes." Recall that a free membrane corresponds to the Neumann boundary value problem. The difficulty in this case is that one cannot apply the Faber-Krahn inequality to nodal domains that are adjacent to $\partial \Omega$ : they

Received by the editors March 24, 2008.

2000 Mathematics Subject Classification. Primary 35B05, 35P99.

Key words and phrases. Laplacian, Neumann boundary conditions, nodal domain. Research partially supported by NSERC and FQRNT.

(C)2008 American Mathematical Society Reverts to public domain 28 years from publication 
have Neumann conditions on a part of their boundary (here and further on we say that a nodal domain $D$ is adjacent to $\partial \Omega$ if length $(\partial D \cap \partial \Omega)>0)$. Later, Pleijel's result was generalized in $\mathrm{Pe}, \mathrm{BM}, \mathrm{Be}$ to higher-dimensional domains with Dirichlet boundary conditions and to compact closed manifolds. However, the case of domains with Neumann boundary conditions remained open.

The purpose of this paper is to prove an analogue of Pleijel's theorem for piecewise analytic planar domains with Neumann boundary conditions. We consider separately the nodal domains that are adjacent to $\partial \Omega$, and the remaining nodal domains having pure Dirichlet conditions on their boundaries. In the latter case, we use the original Pleijel's approach, while in the former we apply an estimate recently obtained by Toth-Zelditch [TZ for the number of boundary zeros of Neumann eigenfunctions. In fact, it follows from [TZ, Theorem 2] that the number of nodal domains adjacent to $\partial \Omega$ is small compared to Courant's bound; see (2.1).

Theorem 1.1. Let $\Omega \subset \mathbb{R}^{2}$ be a piecewise real analytic domain with Neumann boundary conditions. Then the estimate (1.1) holds.

\section{Proof of Theorem 1.1}

Let $m_{k}$ be the number of nodal domains of the eigenfunction $\phi_{k}$ that are adjacent to $\partial \Omega$ and $l_{k}=n_{k}-m_{k}$ be the number of the remaining nodal domains. We show first that

$$
\limsup _{k \rightarrow \infty} \frac{m_{k}}{k}=0
$$

Let $\mathcal{Z}_{k}=\left\{x \in \partial \Omega: \phi_{k}(x)=0\right\}$. According to [TZ, Theorem 2], the number $N_{k}=\operatorname{card}\left(\mathcal{Z}_{k}\right)$ of boundary zeros of the eigenfunction $\phi_{k}$ is bounded by $C \sqrt{\lambda_{k}}$, where $C>0$ is a constant depending only on $\Omega$. It is easy to check that $m_{k} \leq N_{k}+s$, where $s$ is the number of connected components of $\partial \Omega$. Indeed, the set $\partial \Omega \backslash \mathcal{Z}_{k}$ has at most $N_{k}+s$ connected components, and for each connected component $\Gamma \subset \partial \Omega \backslash \mathcal{Z}_{k}$ there exists a unique nodal domain $D$ adjacent to the boundary such that $\Gamma \subset \partial D$ (note that this correspondence is not necessarily one-to-one: the boundary of a nodal domain may contain more than one connected component of $\partial \Omega \backslash \mathcal{Z}_{k}$ ). At the same time, by Weyl's law (see [Ch, p. 9])

$$
\lim _{k \rightarrow \infty} \frac{\lambda_{k}}{k}=\frac{4 \pi}{\operatorname{Area}(\Omega)} .
$$

Therefore, for some constant $C_{1}>0$,

$$
\limsup _{k \rightarrow \infty} \frac{m_{k}}{k} \leq \limsup _{k \rightarrow \infty} \frac{N_{k}+s}{k} \leq \limsup _{k \rightarrow \infty} \frac{C_{1} \sqrt{\lambda_{k}}}{\lambda_{k}}=0,
$$

and this completes the proof of (2.1).

Now let us show that

$$
\limsup _{k \rightarrow \infty} \frac{l_{k}}{k} \leq \frac{4}{j_{1}^{2}}
$$

The proof of this bound is similar to the proof of (1.1); see [Ch, pp. 24-25]. Indeed, let $\tilde{\Omega} \subset \Omega$ be the union of all nodal domains that are not adjacent to $\partial \Omega$. Every such domain $D$ has pure Dirichlet conditions on the boundary, and therefore one can apply the Faber-Krahn inequality:

$$
\lambda_{k}(\Omega) \operatorname{Area}(D)=\lambda_{1}(D) \operatorname{Area}(D) \geq \pi j_{1}^{2} .
$$


Summing up the left- and the right-hand sides over all domains $D$ that are not adjacent to the boundary, we get:

$$
\lambda_{k}(\Omega) \operatorname{Area}(\tilde{\Omega}) \geq l_{k} \pi j_{1}^{2} .
$$

Therefore, taking into account that $\operatorname{Area}(\tilde{\Omega})<\operatorname{Area}(\Omega)$ and using (2.2), one obtains

$$
\limsup _{k \rightarrow \infty} \frac{l_{k}}{k} \leq \limsup _{k \rightarrow \infty} \frac{\lambda_{k}(\Omega) \operatorname{Area}(\tilde{\Omega})}{\pi j_{1}^{2} k} \leq \frac{4}{j_{1}^{2}} .
$$

Since $n_{k}=m_{k}+l_{k}$, putting together (2.1) and (2.3) we complete the proof of the theorem.

Remark 2.1. Theorem 1.1 is proved under the same assumptions as TZ, Theorem 2]. It would be interesting to extend Theorem 1.1 to higher dimensions and to replace piecewise analyticity by a weaker condition. It is likely that the results of [TZ, and hence Theorem 1.1 as well, hold also for domains with mixed Dirichlet-Neumann boundary conditions.

Remark 2.2. It is clear from the proof of (1.1) that this estimate is not sharp for both Dirichlet and Neumann boundary conditions. Indeed, the Faber-Krahn inequality is an equality only for the disk, and nodal domains of an eigenfunction cannot be all disks at the same time. Therefore, a natural problem is to find an optimal constant in (1.1). Motivated by the results of [BGS] we suggest that for any regular bounded planar domain with either Dirichlet or Neumann boundary conditions, $\limsup _{k \rightarrow \infty} \frac{n_{k}}{k} \leq \frac{2}{\pi} \approx 0.636 \ldots$. If true, this estimate (which is quite close to Pleijel's bound) is sharp and is attained for the basis of separable eigenfunctions on a rectangle [P], SS].

\section{REFERENCES}

[Be] P. Berard. Inégalités isopérimétriques et applications. Domaines nodaux des fonctions propres, Séminaire Goulaouic-Meyer-Schwartz (1981-1982), XI.1-XI.9. MR671608 (83m:58078)

[BM] P. Berard and D. Meyer. Inégalités isopérimétriques et applications, Annales Scientifiques de l'École Normale Supérieure Sér. 4, 15, no. 3 (1982), 513-541. MR690651 (84h:58147)

[BGS] G. Blum, S. Gnutzmann, and U. Smilansky. Nodal domain statistics: A criterion for quantum chaos, Phys. Rev. Lett. 88 (2002), 114101-114104.

[Ch] I. Chavel. Eigenvalues in Riemannian geometry, Academic Press, $1984 . \quad$ MR768584 (86g:58140)

[Co] R. Courant. Ein allgemeiner Satz zur Theorie der Eigenfunktionen selbstadjungierter Differentialausdrücke, Nachr. Ges. Göttingen (1923), 81-84.

$[\mathrm{CH}]$ R. Courant and D. Hilbert. Methods of mathematical physics, Vol. 1, Interscience Publishers, New York, 1953.

[EE] D.E. Edmunds and W.D. Evans. Spectral theory and differential operators, Clarendon Press, Oxford, 1987. MR929030 (89b:47001)

[Fa] G. Faber. Beweis, dass unter allen homogenen Membranen von gleicher Fläche und gleicher Spannung die kreisförmige den tiefsten Grundton gibt, Sitzungberichte der mathematisch-physikalischen Klasse der Bayerischen Akademie der Wissenschaften zu München Jahrgang (1923), 169-172.

[Kr] E. Krahn. Über eine von Rayleigh formulierte Minimaleigenschaft des Kreises, Math. Ann. 94 (1924), 97-100. MR1512244

[Pe] J. Peetre. A generalization of Courant's nodal domain theorem, Math. Scand. 5 (1957), 15-20. MR0092917(19:1180a)

[Pl] Å. Pleijel. Remarks on Courant's nodal line theorem, Comm. Pure Appl. Math. 9 (1956), 543-550. MR0080861 (18:315d) 
[SS] U. Smilansky and R. Sankaranarayanan. Nodal domain distribution of rectangular drums, arXiv:nlin/0503002, 1-3.

[TZ] J. Toth and S. Zelditch. Counting nodal lines that touch the boundary of an analytic domain, preprint, arXiv: 0710.0101, 1-27.

Département de Mathématiques et de Statistique, Université de Montréal, CP 6128 Succ. Centre-Ville, Montréal, Quebec H3C 3J7, Canada

E-mail address: iossif@dms.umontreal.ca 